\title{
An Efficient Selective-Repeat ARQ Scheme for Half-duplex Infrared Links under High Bit Error Rate Conditions
}

\author{
Shawkat Shamim Ara, Alam Mohammad Shah and Mitsuji Matsumoto \\ Global Information and Telecommunication Studies \\ Waseda University \\ Tokyo, Japan \\ sunny@asagi.waseda.jp,mithu@fuji.waseda.jp,mmatsumoto@waseda.jp
}

\begin{abstract}
Large window size associated with high-speed infrared links reduces the throughput for IrDA adopted go-back$N$ automatic repeat request (ARQ) scheme of error control rather drastically under high bit error rate (BER) conditions. In this paper, we investigate and analyze a selective-repeat ARQ scheme which operates with a finite receiver buffer and a finite range of sequence numbers in order to achieve higher throughput for half-duplex infrared links at high BER. Simulation results show that it significantly outperforms the existing go-back- $N$ ARQ scheme over a range of BERs including high BER. We also examine the effect of link layer parameters, such as frame length, and physical layer parameters, such as minimum turn around time on the performance of the proposed ARQ scheme.
\end{abstract}

Keywords- Automatic Repeat Request (ARQ) Scheme, Infrared Data Association (IrDA), Infrared Links.

\section{INTRODUCTION}

Infrared (IR) link provides a secure and a promising alternative to radio for wireless indoor applications, be it for terminals or sensors. However, IR systems also suffer from severe noise and disturbances. Since IR systems are subject to large dynamic variations of signal-to-noise ratio (SNR) at the receiver, the system suffers from unacceptably high error rates or loss of connections. Therefore, automatic repeat request (ARQ) error control schemes are used for providing reliable data communications. There are three basic types of ARQ: the stop-and-wait ARQ, the go-back- $N$ ARQ and the selectiverepeat ARQ $[1,2]$. Infrared Data Association (IrDA) has adopted go-back- $N$ (GBN) ARQ scheme for its inherent simplicity. However, the large window size associated with high speed IR links reduces the throughput of this error control scheme rather drastically at high BER conditions and renders the link operation very vulnerable to BER increase $[3,4]$. This inefficiency can be overcome by using ideal selective-repeat (SR) ARQ scheme. However, in the ideal SR ARQ scheme, extensive buffering (theoretically infinite) is required at the receiver to obtain high throughput $[2,3]$.

In this paper, we concentrate on improving the ideal SR ARQ scheme so that it can be efficiently applied in half duplex
IR links for achieving high throughput, particularly at high BER. In recent years, many link layer performance improvements and evaluations have been undertaken to address different IR link issues including minimum turn-around time [5], processing speed [6] and optimum link parameters [4]. In contrast there have not been enough studies on ARQ scheme performance for increasing throughput, especially at high BER. In this work, we investigate a SR ARQ scheme which operates with a finite receiver buffer and a finite range of sequence numbers. The performance of the proposed ARQ scheme is examined for various link parameters and compared with the existing GBN ARQ scheme. Simulation results show that without adapting window size at high BER or using large window size, the proposed ARQ scheme provides significant improvement in the throughput over a range of BERs including high BER. Therefore, it would be applied on IR links for achieving high throughput under high BER conditions.

The paper is outlined as follows. First, we briefly describe the drawbacks of the existing go-back- $N$ (GBN) ARQ scheme on IR links in section 2. Section 3 presents our proposed efficient selective-repeat (ESR) ARQ scheme. In section 4, we present our simulation results for analysis the performance of the proposed ESR ARQ scheme. Finally Section 5 presents our conclusions.

\section{THE EXISTING GO-BACK-N ARQ SCHEME}

In the existing go-back- $N$ (GBN) ARQ scheme on Infrared (IR) links, when a received frame is detected in error, the receiver discards that frame and all the subsequent frames of the window regardless of whether or not they are received successfully and sends negative acknowledgment (NACK) to the transmitter for that error frame. Upon receiving a NACK, the transmitter resends the error frame and $N-1$ succeeding frames that were transmitted during the previous window transmission [7]. As a result, all error free but out of sequence frames of previous transmission has the chance to be in error in the following retransmission. This signifies a waste of transmissions which results in severe deterioration of throughput performance especially in the case of high bit error rate (BER). 


\section{THE PROPOSED EFFICIENT SELECTIVE- REPEAT (ESR) ARQ SCHEME}

For simplicity, we consider the case where the primary station transmits a large amount of information data to the secondary station. The secondary station does not transmit information to the primary; it only sends the acknowledgement of the received frames. However, the proposed scheme can be modified in a straightforward manner for the case where the secondary station also has information data to send. In this work, the maximum window size $(W)$ of 7 frames is considered that has been strongly recommended in [3]. This also allows the proposed scheme to cope with the existing frame structure and adds a small sized receiver buffer and little complexity to the system implementation. But the same idea can also be applicable for larger window sizes under the availability of more bits in the frame and larger buffer at the receiver. Furthermore, we also use some concepts in our proposed scheme that has been proposed for satellite communication systems and modified them to be applicable in half duplex infrared communication systems [8].

In our scheme, we set the range of sequence number of information frames from 0 to $2 W+1$. These numbers will be reused cyclically. The receiver buffer size $2 W+2$ is considered in our scheme.

When an information frame ( $I$-frame) is ready for transmission, it is numbered and stored in the input queue of the transmitter. In normal operation, the transmitter (primary station) of an ESR ARQ scheme sends a window of $I$-frames, polls the receiver (secondary station) by setting the Poll/Final $(P / F)$ bit in the last frame of the window and solicit a response from the receiver.

Since IrDA IR links provide half-duplex communication, the receiver cannot send the acknowledgement of the received frame immediately rather it waits for the completion of current window transmission. If the frame with $P$ bit set is received and as the present case assumes that the secondary station does not sends information, it awaits a minimum turn- around time $(t t a)$ to allow for the hardware recovery latency and transmits the acknowledgement of received frame using supervisory frame with the Poll/Final $(\mathrm{P} / \mathrm{F})$ bit set to pass the transmission control to the primary station.

If no errors are detected in the received frames, the receiver sends positive acknowledgement (ACK) and delivers the frames to the upper layer in consecutive order. In this case, the receiver buffer is empty and the receiver is said to be in the normal state. When a received $I$-frame is detected in error or a received $I$-frame with an out-of-order sequence number is detected, the receiver enters the blocking state. In the blocking state, no frames are delivered to the upper layer. However, the receiver proceeds to check the validity of the subsequent received frames of the window; it stores those frames that are successfully received at proper locations in its buffer, and reserves proper locations in the buffer for the error frames and sends acknowledgement of all the received frames.

\section{A. Multiple Acknowledgements}

The receiver of the ESR ARQ scheme is able to acknowledge all the frames received using the IrDA defined

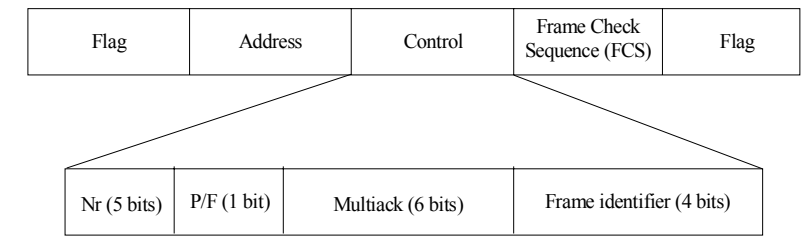

Figure 1. Supervisory frame format for giving Multiple Acknowledgements.

supervisory frame or information frame. [9] For the present case, the receiver sends this multiple acknowledgments using the supervisory frame and the supervisory frames are considered small enough to be error free. We rearrange the frame structure of the existing supervisory frame to cope with our proposed scheme for giving this multiple acknowledgements (see Fig. 1).

In this frame, the $N r$ field is set to the sequence number of next expected frame, i.e., the sequence number of first error frame. So, the $N r$ field acknowledges all correctly received $I$ frames having frame sequence number less than $N r$. The Multiack field reports the reception status of all frames having sequence number greater than $\mathrm{Nr}$ using the bit map information i.e., by inserting " 1 " (ACK) for successfully received frame and " 0 " (NACK) for erroneously received frame in the corresponding bit positions.

For the case, where the secondary station also has information to send, it can send this multiple acknowledgements using the existing $I$-frame.

\section{B. Retransmission strategy}

Upon receiving NACK, the transmitter of the ESR ARQ scheme retransmits only the error frames, not the out of sequence correct frames and new frames to form a window transmission. The retransmission procedure is described as follows (see Fig. 2).

At first the transmitter resends the first error frame. Then, for the frame following the first error frame in the previous transmission, if a NACK is received, the transmitter resends
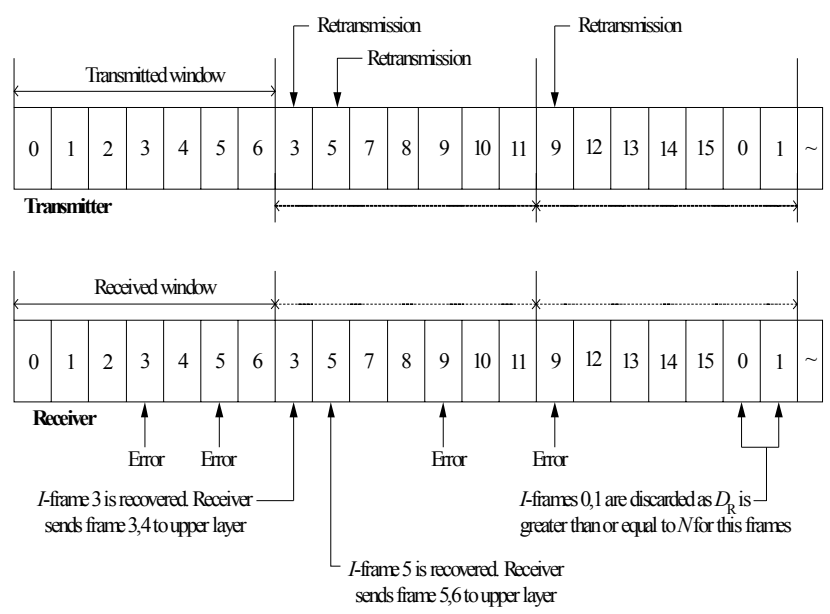

Figure 2. Proposed Efficient Selective-Repeat (ESR) ARQ scheme with window size $(N)$ of 7 frames. 


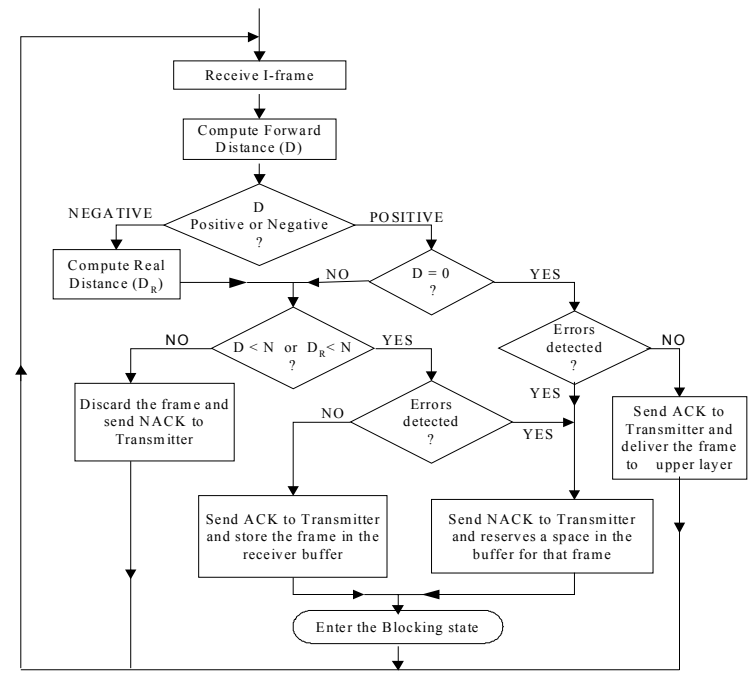

Figure 3. Receiver operation during Recovery procedure.

that error frame. If an ACK is received for that frame, it sends the new $I$-frame from its input queue.

If the receiver could receive some frames in correct sequence and error free in the previous transmission attempt, the transmitter sends some more new frames to form a complete $N$ (window size) frames transmission.

The main idea used in this scheme is to resend only error frames and send some new frames during retransmission. When the receiver recovers the first erroneous frame successfully, it can provide buffer space for the new frames. This increases the probability of receiving more new frames at the receiver during the retransmission period and thus offering significant improvement in the throughput.

Furthermore, correctly received out of sequence frames of the previous transmission are not retransmitted in this scheme. So, the probability of being error of that frame in the following retransmission is also reduced.

\section{Recovery Procedure}

As soon as the first error $I$-frame is successfully recovered, the receiver starts to send that frame and the subsequent errorfree frames (if any), which are held up in the receiver buffer during the blocking state to the upper layer (see Fig. 2). The receiver will continue this process until the next error frame is encountered. If after the recovery of the first error frame, all the frames in the receiver buffer are released and all the reserved locations are freed, it works like that in normal state. However, if there are still some reserved locations in its buffer or not all the frames in the buffer can be released to the upper layer, then it will continue its recovery process until all the error frames are recovered and also confirm that buffer overflow never occurs. Now we describe the recovery procedure.

When an I-frame is received, the receiver checks its validity and also computes its forward distance with respect to the next expected frame, denoted $D$, which is defined as follows:

$D=s-e$

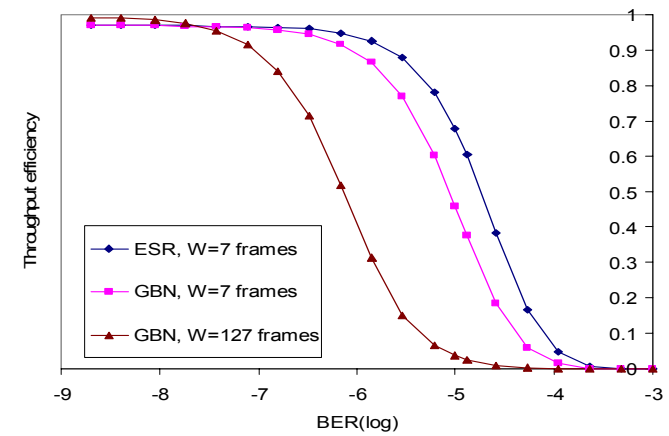

Figure 4. Throughput efficiency versus BER for the GBN and the proposed ESR ARQ scheme with $C=16 \mathrm{Mb} / \mathrm{s}, t t a=0.1 \mathrm{~ms}, L=2 \mathrm{~KB}$

where $\mathrm{s}$ is the sequence number of current received $I$-frame and $\mathrm{e}$ is sequence number of the next expected frame. The forward distance $D$ might have either positive value or negative value.

If the forward distance $D$ of the current received frame is negative, the receiver computes its real distance $D_{R}$ by adding forward distance $D$ with maximum sequence number $(2 W+2)$. The real distance is defined as follows:

$D_{R}=D+(2 W+2)$.

The detailed operation of the receiver during the recovery procedure is illustrated in Fig. 3.

\section{THROUGHPUT ANALYSIS}

To examine the performance of the proposed ESR ARQ scheme and the effect of different link parameters on it, a set of simulation runs was performed using the OPNET ${ }^{\mathrm{TM}}$ simulation package [10]. Our OPNET simulator closely follows all the behaviors of primary and secondary stations, including transmission times, turn around delays and transmission errors.

Fig. 4 compares the throughput efficiency of the existing GBN and proposed ESR ARQ scheme for link data rate $(C)$ $16 \mathrm{Mb} / \mathrm{s}$ with minimum turn-around time $(t t a) 0.1 \mathrm{~ms}$, and $I-$ frame message data length $(L) 2 \mathrm{~KB}$. It shows that the throughput is significantly increased for a range of BER values (from $10^{-6}$ to $10^{-4}$ ) including high BER if the proposed ARQ scheme is employed. For low BER it provides same throughput efficiency (0.97) as that using the GBN ARQ scheme with window size of 7 frames $(W=7)$. However, if the BER increases it provides a significant improvement in the throughput compared to the GBN scheme with $W=7$. This is because in our proposed scheme, all the error free but out of sequence frames following the first erroneous frame are stored in the buffer and are not retransmitted during the retransmission. It also shows that how the large window size $(W=127)$ associated with high speed IR links reduces the throughput of the existing GBN scheme rather drastically at high BER conditions and renders the link operation very vulnerable to BER increase. In the case of our scheme by reducing window size to 7 frames $(W=7)$, an excellent performance is observed both in terms of throughput and robustness to BER increase. Thus, from our simulation results, 


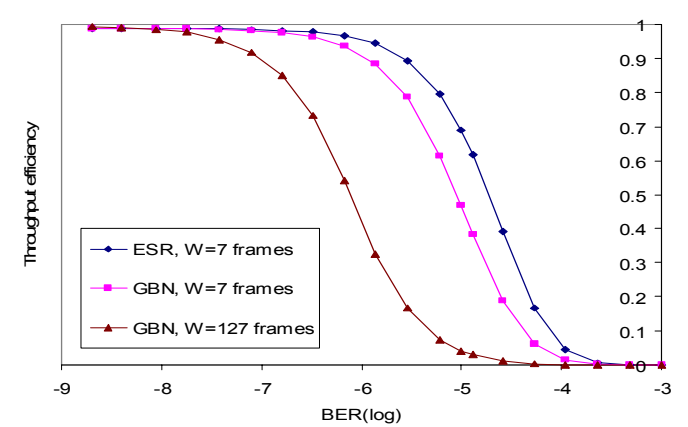

Figure 5. Throughput efficiency versus BER for the GBN and the proposed ESR ARQ scheme with $C=4 \mathrm{Mb} / \mathrm{s}, t t a=0.1 \mathrm{~ms}, L=2 \mathrm{~KB}$.

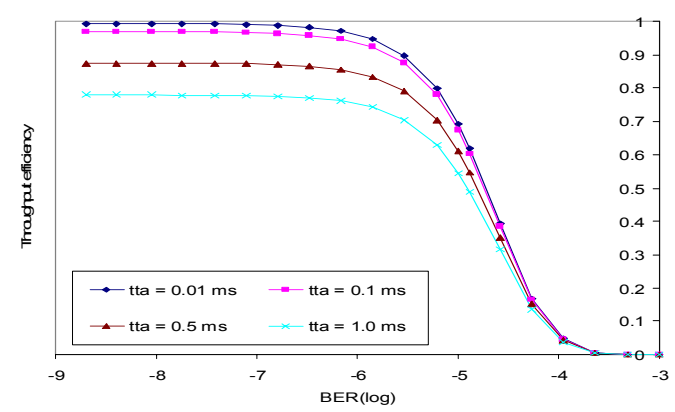

Figure 6. Throughput efficiency versus BER for the proposed ESR ARQ scheme with $C=16 \mathrm{Mb} / \mathrm{s}, W=7$ frames, $L=2 \mathrm{~KB}$.

it is obvious that large window sizes are not necessary, and a much smaller window size defined in current IrDA, which is 7 , is sufficient for having high throughput efficiency over a range of BERs including high BER.

The same comparison for a $4 \mathrm{Mb} / \mathrm{s}$ link with $t t a=0.1 \mathrm{~ms}, L=2 \mathrm{~KB}$ is shown in Fig. 5. This also confirms that the proposed ESR ARQ scheme using window size of 7 frames $(W=7)$ significantly outperforms the existing GBN ARQ scheme.

Fig. 6 plots throughput efficiency versus link BER. It examines the effect of reducing turn-around time $(\mathrm{tta})$ on the throughput efficiency of the ESR ARQ scheme for a $16 \mathrm{Mb} / \mathrm{s}$ link with $W=7$ frames, $L=2 \mathrm{~KB}$. It is revealed that throughput is increased significantly when turn-around time is reduced and an excellent performance is observed by reducing turn-around time $t t a$ to $0.1 \mathrm{~ms}$. Taking further advantage of turn-around time decrease $(t t a=0.01 \mathrm{~ms})$ results in a slightly better performance for low BER but for high BER it provides same throughput as that of using turn-around time $0.1 \mathrm{~ms}$. So, small minimum turnaround delay (tta=0.1 ms) should be used for achieving maximum throughput.

The effect of increasing frame length $(L)$ on the throughput efficiency of the proposed ESR ARQ scheme for a $16 \mathrm{Mb} / \mathrm{s}$ link with $t t a=0.1 \mathrm{~ms}$ and $W=7$ frames is shown in Fig. 7. Frame length increase results in slight throughput increase for low BER but renders the link vulnerable to BER increase.

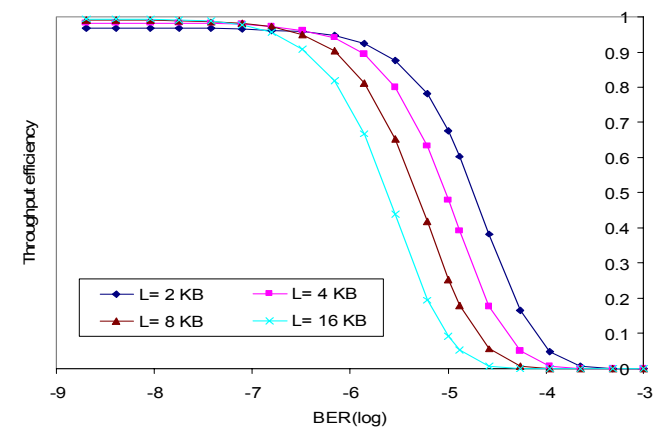

Figure 7. Throughput efficiency versus BER for the proposed ESR ARQ scheme with $C=16 \mathrm{Mb} / \mathrm{s}, t t a=0.1 \mathrm{~ms}, W=7$ frames.

\section{CONCLUSIONS}

In this paper, we have investigated an efficient selectiverepeat (ESR) ARQ scheme which operates with a finite receiver buffer and a finite range of sequence numbers over half duplex IR links. A multiple acknowledgement scheme that utilizes the existing supervisory frame or information frame to provide acknowledgements of all the received frames is employed in the ESR ARQ scheme. Simulation results are presented which shows that the proposed ARQ scheme significantly outperforms the existing go-back- $N$ (GBN) ARQ scheme, particularly for links with high BER. The significance of the physical layer minimum turn-around time and other link layer parameters on throughput performance of the proposed scheme are also explained. We also explored that without adapting window size at high BER or using large window size, the proposed ARQ scheme using window size of 7 frames provides a significant improvement in the throughput, particularly at high BER. Thus, large window sizes are not necessary, and a much smaller window size defined in current IrDA, which is 7 , is sufficient for achieving high throughput efficiency over a range of BERs including high BER. Therefore, it would be applied on half-duplex IR links for achieving high throughput over a range of BERs including high BER, as it gives substantial improvement in the throughput efficiency with little additional complexity in system implementation.

\section{REFERENCES}

[1] H. O. Burton and D. D. Sullivan, "Errors and Error Control," Proc. IEEE., vol.60, pp. 1293-1303, Nov.1972.

[2] S. Lin, D. Costello and M. Miller, "Automatic- Repeat- Request Error Control Schemes," IEEE Comm. Mag., vol.22, no.12, pp.5-17, Dec. 1984.

[3] T. Ozugur, M. Naghshineh and P. Kermani, "Comparison of go-back-N and selective reject ARQ modes of HDLC over half-duplex and fullduplex IR links and the effects of window size and processor speed in utilization," IEEE International Symposium on Personal, Indoor and Mobile Radio Communications, vol.2, pp.708-712, Sept.1998.

[4] V. Vitsas and A. C. Boucouvalas, "Optimization of IrDA IrLAP Link Access Protocol," IEEE Trans. Wireless Commun., vol.2, no.5, pp.926938, Sep.2003.

[5] A. C. Boucouvalas, V.Vitsas, "100 Mb/s IrDA Protocol Performance Evaluation", Proceedings of IASTED International Conference on Wireless and Optical Communications (WOC 2001), pp.49-57, 2001. 
[6] P. Chatzimisios and A. C. Boucouvalas "IrLAP IrDA Protocol Throughput Dependence on Processor Speed" International Symposium on CSNDSP 2002, Staffordshire University, pp. 272-275, July 2002.

[7] P. Barker and A. C. Boucouvalas, "Performance Modeling of the IrDA Protocol for Infrared Wireless Communications," IEEE Commun. Mag., vol. 36, no.12, pp.113-117, Dec. 1998.

[8] Miller and S. Lin, "The Analysis of Some Selective-Repeat ARQ Schemes with Finite Receiver Buffer," IEEE Trans. Commun., vol. COM-29, no. 9, pp.1307-1315, Sep. 1981.

[9] IrDA, Serial Infrared Link Access Protocol (IrLAP), Version 1.1 (Infrared Data Association, 1996).

[10] OPNET $^{\mathrm{TM}}$ modeller, MIL3 Inc., 3400 International Drive NW, Washington DC 20008, USA. 